\title{
Oscillations of electrostatically trapped particles
}

\author{
V. C. LIu and R. J. Hong \\ Department of Aerospace Engineering, The University of Michigan, Ann Arbor, \\ Mich. 48104, U.S.A.
}

(Received 17 February 1969; in Revised form 5 May 1969)

\begin{abstract}
A kinetic theory of longitudinal ionic oscillations is presented which pertains to an earlier hypothesis that low frequency plasma oscillations monitored in the wake of Ariel I Satellite are due to ions trapped in the potential trough behind a moving satellite. The treatment is one-dimensional. A self-consistent potential distribution in the trough is obtained from a linearized analysis, suitable for a potential trough of small depth. Dispersion relation of the plasma oscillations for the trapped particles are obtained. The conditions for instability of the oscillation are given. The present analysis further confirms the earlier hypothesis as stated above.
\end{abstract}

\section{INTRODUCTION}

WHEN a large conducting body with a negative surface potential moves at a mesothermal spcod* in a rarefied plasma, e.g. an artificial satellite orbiting in the upper ionosphere, a negative potential valley is found (LIU and JEw, 1967; LIU, 1969b) to develop in the near wake behind the moving body. It was later shown theoretically (LrU, 1967; LrU, 1969a) that ions should be trapped in the potential valley where they perform longitudinal oscillations of low frequencies. It was demonstrated (LIU, 1967; LIU, 1969a) that these ion oscillations might account for the low frequency fluctuations found in the wake of Ariel satellite I when Langmuir probe was used to measure the electron density and temperature of the ionosphere. The connection between the above mentioned ion oscillations and the high frequency oscillations found in the ionograms of the top sounder Alouette satellites has been discussed (LIU, 1967; LIU, 1969a).

It should be recognized that the discussions presented by LIU (1967) and LIU (1969a) were developed on the basis of some crude approximations, e.g. the dispersion relation of longitudinal ion oscillations in an inhomogeneous electrostatic field was obtained using essentially Langmuir-Tonks derivation for a homogeneous plasma with minimum modification for field inhomogeneity; thereby neglecting the microscopic velocity distribution of the particles. These previous analyses also left unanswered the question of wave amplification and damping which are crucial in the problem particularly when there is coupling between the oscillations of high and low frequencies. It is to provide a microscopic or kinetic analysis of the ion oscillations hence to present a more convincing discussion on the plasma behavior of the electrostatically trapped particles that the present study is ascribed.

Consider the symmetric potential valley in the near wake of a satellite (LIv and $\mathrm{JEw}, 1967$ ). A meaningful one-dimensional model can be constructed if the discussion is restricled to the potential discribution along the line of symmetry in the wake. Since our interest is primarily on the oscillations of the trapped ions whose Larmor radii in question are much larger than the width of the potential valley we

* A mesothermal speed refers to a value which is much larger than the thermal speed of ions and yet much smaller than that of electrons. 
may ignore the geomagnetic field effect in constructing an idealized model which has a potential distribution self consistent with Poisson and collisionless Boltzmann equations (Chapman and Cowling, 1939; GinzBURG, 1961).

$$
\begin{gathered}
\frac{\partial F}{\partial t}+C_{x} \frac{\partial F}{\partial x}-\frac{e}{M} \frac{\partial \varphi}{\partial x} \frac{\partial F}{\partial C_{x}}=0 \\
\frac{\partial f}{\partial t}+C_{x} \frac{\partial f}{\partial x}+\frac{e}{m} \frac{\partial \varphi}{\partial x} \frac{\partial f}{\partial C_{x}}=0 \\
\nabla^{2} \varphi=-4 \pi e\left(\int_{-\infty}^{\infty} F^{\prime} d C_{x}-\int_{-\infty}^{\infty} f d C_{x}\right) .
\end{gathered}
$$

Note that $F$ and $f$ denote ions and electrons distribution function respectively. As stated before, a large conducting body with a negative surface potential moves at a mesothermal speed, $A \ll V_{0} \ll a$, where $V_{0}$ is body velocity; $A$, $a$, the mean ion and electron thermal speed respectively, in a fully ionized rarefied plasma. Let $C_{x}=$ $v+V_{0}$, where $v$ denotes the thermal velocity, equations (1.1-1.3) become

$$
\begin{gathered}
\frac{\partial F}{\partial t}+\left(v+V_{0}\right) \frac{\partial F}{\partial x}-\frac{e}{M} \frac{\partial \varphi}{\partial x} \frac{\partial F}{\partial v}=0 \\
\frac{\partial f}{\partial t}+\left(v+V_{0}\right) \frac{\partial f}{\partial x}+\frac{e}{m} \frac{\partial \varphi}{\partial x} \frac{\partial f}{\partial v}=0 \\
\nabla^{2} \varphi=-4 \pi e\left(\int_{-\infty}^{\infty} F d v-\int_{-\infty}^{\infty} f d v\right) .
\end{gathered}
$$

The present study, therefore, consists of two parts: (1) the steady state solution for the self-consistent potential distribution in the trough; $(2)$ the dispersion relation of the longitudinal plasma oscillations in the trough. In part (2) the formulation is valid for the full range of oscillation frequencies; the solution for the low frequency oscillations $\left(\kappa / \kappa_{D} \ll 1\right)$ only is given here. From the results of dispersion relation we are able to discuss the damping and amplification of the disturbances as functions of the characteristics of ambient plasma and the potential trough.

\section{Steady State Distribution of Plasma (in the Trough)}

Consider a one-dimensional potential trough along the $x$-axis with its origin at the minimum potential point (see Fig. 1). It is assumed that the trough symmetric with respect to its midpoint $(x=0)$ is filled with fully ionized plasma having ion density $n_{i}(x)$ and electron density $n_{e}(x)$. It is assumed that the potential distribution in the trough is truncated at points $A$ and $B$, where the potential $\varphi \approx 0$ and the ion density is $n_{i 0}$; electron density $n_{e 0}$. It is postulated that the trough is surrounded by the ambient plasma of singly-charged ions and electrons of temperatures $T_{i}$ and $T_{e}$. It is of importance to provide a bi-thermal state which characterizes the daytime characteristics of upper ionosphere. It is assumed that the plasma is collisionfree which again is valid for the tenuous upper ionosphere.

Under the steady state, the distributions of ions, $F_{0}(x, v)$ and electrons, $f_{0}(x, v)$ 


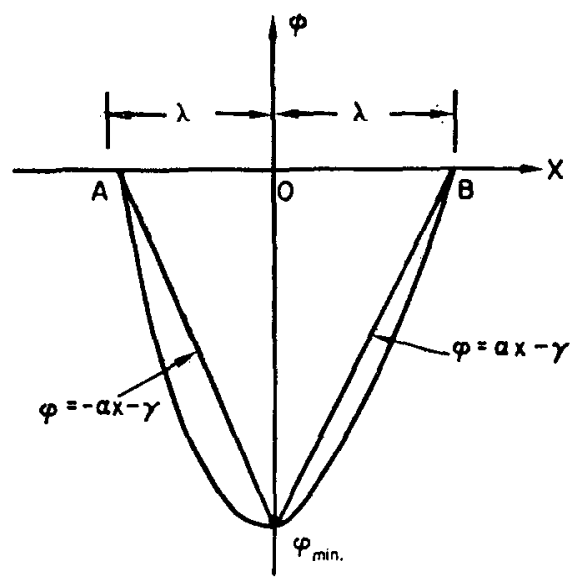

Fig. 1. Potential distributions in the trough.

and electrostatic potential, $\phi_{0}(x)$, are governed by the following set of equations:

$$
\begin{gathered}
\left(v+V_{0}\right) \frac{\partial F_{0}}{\partial x}-\frac{e}{M} \frac{\partial \varphi_{0}}{\partial x} \frac{\partial F_{0}}{\partial v}=0 \\
\left(v+V_{0}\right) \frac{\partial f_{0}}{\partial x}+\frac{e}{m} \frac{\partial \varphi_{0}}{\partial x} \frac{\partial f_{0}}{\partial v}=0 \\
\nabla^{2} \varphi_{0}=-4 \pi e\left[\int_{-\infty}^{\infty} F_{0}(x, v) d v-\int_{-\infty}^{\infty} f_{0}(x, v) d v\right] .
\end{gathered}
$$

The Lagrangian equation subsidiary to (2.1) can be written

$$
\frac{d x}{v+V_{0}}=\frac{d v}{-\frac{e}{M} \frac{\partial \varphi_{0}}{\partial x}}
$$

which is integrated to

$$
\frac{1}{2} M\left(v+V_{0}\right)^{2}+e \varphi_{0}=\text { const. }
$$

Thus the general solution of (2.1) becomes

$$
F_{0}=F_{0}\left[\frac{1}{2} M\left(v+V_{0}\right)^{2}+e \varphi_{0}\right]=\frac{n_{i 0}}{\sqrt{ }(\pi) A} \exp \left[-\frac{\left(v+V_{0}\right)^{2}}{A^{2}}-\frac{e \varphi_{0}}{k T_{i}}\right]
$$

the equilibrium distribution of Maxwell-Boltzmann, where $A^{2} \equiv 2 k T_{i} / M$ and $A$ denotes the thermal speed of ions.

Similarly we have the steady state electron distribution, from the use of (2.2)

$$
f_{0}=\frac{n_{e 0}}{\sqrt{ }(\pi) a} \exp \left[-\frac{\left(v+V_{0}\right)^{2}}{a^{2}}+\frac{e \varphi_{0}}{k T_{s}}\right]
$$

also the Maxwell-Boltzmann distribution where $a^{2} \equiv 2 k T_{\mathrm{s}} / m$ with $a$ denoting the thermal speed of electrons. 
Substitution of (2.6) and (2.7) into (2.3) leads to

$$
\nabla^{2} \varphi_{0}=-4 \pi e\left(n_{i 0} \exp \left[-\frac{e \varphi_{0}}{k T_{i}}\right]-n_{e 0} \exp \left[\frac{e \varphi_{0}}{k T_{\ell}}\right]\right) .
$$

Here we introduce another crucial assumption for simplification of analysis: $e \varphi_{0} / k T_{i} \ll 1$ and $e \varphi_{0} / k T_{e} \ll 1^{*}$ which imply that both thermal energies $k T_{i}$ and $k T_{e}$ are large compared with the electric potential energy of the particles. Under the present assumption, (2.8) can be linearized to

$$
\nabla^{2} \varphi_{0}=\varphi_{0}\left(\lambda_{D}{ }^{-2}+\lambda_{d}{ }^{-2}\right)-k T_{i} / e \lambda_{D}{ }^{2}+k T_{e} / e \lambda_{d}{ }^{2}
$$

after dropping the second and higher order terms of $e \varphi_{0} / k T_{i}$ and $e \varphi_{0} / k T_{e}$; note that

$$
\lambda_{D}{ }^{2}=k T_{i} /\left(4 \pi e^{2} n_{i 0}\right) ; \quad \lambda_{d}^{2}=k T_{e} /\left(4 \pi e^{2} n_{e 0}\right)
$$

where $\lambda_{D}, \lambda_{d}$ denote the Debye shielding length for ions and electrons respectively.

It can be shown that (2.9) is satisfied by

$$
\varphi_{0}=b \cosh \frac{x}{\lambda^{\prime}}+\frac{\lambda_{d}{ }^{2} k T_{i}}{e\left(\lambda_{d}^{2}+\lambda_{D}{ }^{2}\right)}-\frac{\lambda_{D}{ }^{2} k T_{e}}{e\left(\lambda_{d}^{2}+\lambda_{D}{ }^{2}\right)}
$$

where $\lambda^{\prime} \equiv \lambda_{D} \lambda_{d} /\left(\lambda_{d}^{2}+\lambda_{D}\right)^{1 / 2}$ and $b$, a constant.

To estimate the truncated distance $X_{B}$, we obtain from (2.11) by putting $\varphi_{0}=0$ and $\cosh X \mid \lambda^{\prime} \approx 1+X_{B}{ }^{2} / 2 \lambda^{\prime 2}+\ldots$ assuming $|X| \lambda^{\prime} \mid \ll 1$ also $e b / k T_{i} \ll 1$ and $e b / k T_{e} \ll 1$ which is justifiable,

$$
X_{B}=\lambda_{D}\left(1+\frac{T_{i}}{T_{e}} \frac{n_{e 0}}{n_{i 0}}\right)\left\{\frac{2 k T_{i}}{e b}\left(\frac{n_{e 0}}{n_{i 0}}-1\right)\right\}^{1 / 2}
$$

and $X_{A}=-X_{B}$. Notice that for a negative potential trough (see Fig. 1) $n_{e 0} / n_{i 0}>1$, for ion trapping (BoHM and Gross, 1949; Bernstein, GreEne and Kruskal, 1957), which always results in a positive value in the bracket of (2.12). To simplify the analysis, we choose two straight lines which connect two terminal points in $x$ axis to $\varphi_{\min }$ point respectively, to replace parabolic variation for potential trough distribution. Thus the steady state potential distribution in the trough can be expressed as

where

$$
\varphi_{0}= \pm \alpha x-\gamma
$$

$$
\alpha=\frac{b}{\lambda_{D}}\left[\frac{k T_{i}}{2 e b}\left(\frac{n_{e 0}}{n_{i 0}}-1\right)\right]^{1 / 2}\left(1+\frac{T_{i}}{T_{e}} \frac{n_{e 0}}{n_{i 0}}\right)^{-2}
$$

and

$$
\gamma=\frac{k T_{i}}{e}\left(\frac{n_{e 0}}{n_{i 0}}-1\right)\left(1+\frac{T_{i}}{T_{e}} \frac{n_{e 0}}{n_{i 0}}\right)^{-1} .
$$

* Recent work by Anderson and Arthurs (1968) who solved the Poisson equation (2.8) without these low-potential restrictions by using a complementary variational principle gives a solution which has the same functional form as our solution (2.11) except with different constants. In view of the above cited reference, these low-potential assumptions can be removed provided appropriate adjustment for the parameters $\alpha$ and $\gamma$ in the linear approximation (2.13) are made accordingly. 


\section{Transient State Plasma Perturbations}

When the plasma particles in the potential trough are disturbed, they must so deviate from the respective steady state such that the resultant distributions $F, f$ and $\varphi$ must satisfy the Poisson equation (1.6) and the time-dependent collisionless Boltzmann equation (1.4) and (1.5) along with prescribed boundary conditions.

We shall start with the self-consistent system of equations for longitudinal electrostatic oscillations suitable for three-dimensional problems. Let

$$
\left.\begin{array}{c}
F=F_{0}(\mathbf{x}, \mathbf{v})+F_{1}(\mathbf{x}, \mathbf{v}, t) \\
f=f_{0}(\mathbf{x}, \mathbf{v})+f_{1}(\mathbf{x}, \mathbf{v}, t) \\
\varphi=\phi_{0}(\mathbf{x})+\varphi_{1}(x, t)
\end{array}\right\}
$$

where $F_{1} \ll F_{0}, f_{1} \ll f_{0}$ and $\varphi_{1} \ll \varphi_{0}$. When (3.1) is substituted into the Poisson equation (1.6) and the time-dependent collisionless Boltzmann equation (1.4) and (1.5), we obtain, after the use of (2.1), (2.2) and (2.3), the first order system of equations:

$$
\begin{gathered}
\frac{\partial F_{1}}{\partial t}+\left(\mathbf{v}+\mathbf{V}_{0}\right) \cdot \frac{\partial F_{1}}{\partial \mathbf{x}}-\frac{e}{M} \frac{\partial \varphi_{1}}{\partial \mathbf{x}} \cdot \frac{\partial F_{0}}{\partial \mathbf{v}}-\frac{e}{M} \frac{\partial \varphi_{0}}{\partial \mathbf{x}} \cdot \frac{\partial F_{1}}{\partial \mathbf{v}}=0 \\
\frac{\partial f_{1}}{\partial t}+\left(\mathbf{v}+\mathbf{V}_{0}\right) \cdot \frac{\partial f_{1}}{\partial \mathbf{x}}+\frac{e}{m} \frac{\partial \varphi_{1}}{\partial \mathbf{x}} \cdot \frac{\partial f_{0}}{\partial \mathbf{v}}+\frac{e}{m} \frac{\partial \varphi_{0}}{\partial \mathbf{x}} \cdot \frac{\partial f_{1}}{\partial \mathbf{v}}=0 \\
\nabla^{2} \varphi_{1}=-4 \pi e\left(\int_{-\infty}^{\infty} F_{1} d \mathbf{v}-\int_{-\infty}^{\infty} f_{1} d \mathbf{v}\right)
\end{gathered}
$$

along with the following boundary conditions

$$
F_{1}=f_{1}=0 \quad \text { and } \quad \varphi_{0}=\psi_{1}=0 \quad \text { at }|x|=\lambda .
$$

Applying (3.5) to (3.2) and (3.3) with the following Laplace transform with respect to time and Fourier (finite) transforms with respect to space

$$
\left.\begin{array}{rl}
\bar{F}_{1}(\omega, \kappa ; \mathbf{x}, \mathbf{v}) & =\int_{-\lambda}^{\lambda} \int_{0}^{\infty} F_{1}(t, \mathbf{x}, \mathbf{v}) e^{i \omega t-i \kappa \cdot\left(\mathbf{x}^{\prime}-\mathbf{x}\right)} d t d \mathbf{x}^{\prime} \\
\bar{\varphi}_{1}(\omega, \kappa ; \mathbf{x}) & =\int_{-\lambda}^{\lambda} \int_{0}^{\infty} \varphi_{1}\left(t, \mathbf{x}^{\prime}\right) e^{i \omega t-i \kappa \cdot\left(\mathbf{x}^{\prime}-\mathbf{x}\right)} d t d \mathbf{x}^{\prime} \\
\bar{f}_{1}(\omega, \kappa ; \mathbf{x}, \mathbf{v}) & =\int_{-\lambda}^{\lambda} \int_{0}^{\infty} f_{1}\left(t, \mathbf{x}^{\prime}, \mathbf{v}\right) e^{i \omega t-i \kappa \cdot\left(\mathbf{x}^{\prime}-\mathbf{x}\right)} d t d \mathbf{x}^{\prime}
\end{array}\right\}
$$

and simplifying, we obtain the transformed collisionless Boltzmann equation for ions as follows:

$$
\begin{aligned}
\frac{e \alpha}{M} \frac{\kappa}{|\kappa|} \cdot \frac{\partial \bar{F}_{1}}{\partial \mathbf{v}}-i\left[\kappa \cdot\left(\mathbf{v}+\mathbf{V}_{0}\right)-\omega\right] \vec{F}_{1}-\overline{g_{0 i}}+\frac{e}{M} \frac{\partial \tilde{F}_{0}}{\partial \mathbf{v}} \\
\quad \times\left\{i\left[\left(1+\tilde{\gamma}_{i}\right) \kappa+\frac{\kappa}{|\kappa|} \tilde{\alpha}_{i}(-i+\kappa \cdot \mathbf{x}) \bar{\phi}_{1}-\frac{\kappa}{|\kappa|} \tilde{\alpha}_{i} \kappa \cdot \frac{\partial \phi_{1}}{\partial \kappa}\right\}=0\right.
\end{aligned}
$$


where

$$
\begin{aligned}
\vec{g}_{0 i} & =\int_{-\lambda}^{\lambda} \int_{0}^{\infty} F_{1}\left(t=0, \mathbf{x}^{\prime}, \mathbf{v}\right) e^{i \omega t-i \kappa^{*}\left(\mathbf{x}^{\prime}-\mathbf{x}\right)} d t d \mathbf{x}^{\prime} \\
\tilde{F}_{\mathbf{0}}\left(\mathbf{v}+\mathbf{V}_{0}\right) & =\frac{n_{i 0}}{\sqrt{ }(\pi) A} e^{-\left(\mathbf{v}+\nabla_{0}\right)^{2} / A^{2}} \\
\tilde{\alpha}_{i} & =\frac{e \alpha}{k T_{i}} \quad \text { and } \quad \tilde{\gamma}_{i}=\frac{e \gamma}{k T_{i}} .
\end{aligned}
$$

For one-dimensional case, let $\mathbf{V}_{0} \| \kappa$, the direction of propagation and $\beta$ the angle between vectors $\mathbf{v}$ and $\kappa$, then (3.7) becomes (Bernstein, 1958; LIU and Hung, 1968 ), the direction of $\mathrm{x}$ being aligned with $x$-axis,

$$
\begin{aligned}
\frac{\partial \bar{F}_{1}}{\partial \beta}+i \frac{M v}{e \alpha} & {\left[\kappa v \cos \beta-\left(\omega-\kappa V_{0}\right)\right] \bar{F}_{1}-\frac{M v}{e \alpha} \bar{g}_{0 i} } \\
& \quad-\frac{v}{\alpha} \cos \beta \frac{\partial \tilde{F}_{0}}{\partial v}\left\{i\left[\left(1+\tilde{\gamma}_{i}\right) \kappa+\tilde{\alpha}_{i}(-i+\kappa x)\right] \bar{\phi}_{1}-\tilde{\alpha}_{1} \kappa \frac{\partial \bar{\phi}_{1}}{\partial \kappa}\right\}=0 .
\end{aligned}
$$

This is a first order ordinary differential equation. The integrating factor can be expressed as

$$
\exp \int_{\beta}^{\beta^{\prime}}\left\{-i \frac{M v}{e \alpha}\left[\kappa v \cos \beta-\left(\omega-\kappa V_{0}\right)\right]\right\} d v
$$

Multiplying (3.9) by the above integrating factor makes the equation an exact differential, which can then be integrated. The solution is thus obtained as

$$
\begin{aligned}
F_{1}=\frac{v}{\alpha} \frac{\partial \tilde{F}_{0}}{\partial v}\left\{i\left[\left(1+\tilde{\gamma}_{i}\right) \kappa+\tilde{\alpha}_{i}(-i+\kappa x)\right] \bar{\phi}_{1}-\tilde{\alpha}_{i} \kappa \frac{\partial \bar{\phi}_{1}}{\partial \kappa}\right\} \\
\times \exp \left\{i \frac{M v}{e \alpha}\left[\kappa v \sin \beta-\left(\omega-\kappa V_{0}\right) \beta\right]\right\} \int_{\infty}^{\beta} \cos \beta^{\prime} \\
\quad \times \exp \left\{-i \frac{M v}{e \alpha}\left[\kappa v \sin \beta^{\prime}-\left(\omega-\kappa V_{0}\right) \beta^{\prime}\right]\right\} d \beta^{\prime}+\bar{G}_{0 i}
\end{aligned}
$$

where

$$
\begin{aligned}
\bar{G}_{0 i}=-\frac{M v}{e \alpha} \exp \left\{i \frac{M v}{e \alpha}\left[\kappa v \sin \beta-\left(\omega-\kappa V_{0}\right)\right]\right\} \int_{\infty}^{\beta} \bar{g}_{0 i} \\
\quad \times \exp \left\{-i \frac{M v}{e \alpha}\left[\kappa v \sin \beta^{\prime}-\left(\omega-\kappa V_{0}\right) \beta^{\prime}\right]\right\} d \beta^{\prime} .
\end{aligned}
$$

Making use of the identities

(3.10) becomes

$$
\left.\begin{array}{rl}
\cos \beta e^{-i z \sin \beta} & =\sum_{n} \frac{n}{z} J n(z) e^{-i n \beta} \\
e^{i z \sin \beta} & =\sum_{n} J n(z) e^{i n \beta}
\end{array}\right\}
$$

$$
\begin{aligned}
F_{1}=\frac{e}{M} \sum_{m} \sum_{n} \frac{n e \alpha}{M \kappa \omega^{\prime} v} & \frac{e^{i \beta(m-n)}}{v-\frac{n e \alpha}{M \omega^{\prime}}} \frac{\partial \tilde{F}_{0}}{\partial v} J n\left(\frac{M \kappa v^{2}}{e \alpha}\right) J m\left(\frac{M \kappa v^{2}}{e \alpha}\right) \\
& \times\left\{\left[\left(1+\tilde{\gamma}_{i}\right) \kappa+\tilde{\alpha}_{i}(-i+\kappa x)\right] \bar{\phi}_{1}+i \tilde{\alpha}_{i} \kappa \frac{\partial \bar{\phi}_{1}}{\partial \kappa}\right\}+\bar{G}_{0 i}
\end{aligned}
$$


where $J_{n}$ and $J_{m}$ denote the Bessel function of the first kind for order $n$ and $m$ respectively. $\omega^{\prime}=\omega-\kappa V_{0}$ is the Doppler frequency (StIx, 1962).

Similarly, we have the electron parts as follows:

$$
\begin{aligned}
\bar{f}_{1}=-\frac{e}{m} \sum_{m} \sum_{n} \frac{n e \alpha}{m \kappa \omega^{\prime} v} \frac{e^{i \beta(m-n)}}{v-\frac{n \alpha e}{m \omega^{\prime}}} \frac{\partial \tilde{f}_{0}}{\partial v} J n\left(\frac{m \kappa v^{2}}{e \alpha}\right) J m\left(\frac{m \kappa v^{2}}{e \alpha}\right) \\
\quad \times\left\{\left[\left(1-\tilde{\gamma}_{e}\right) \kappa-\tilde{\alpha}_{e}(-i+\kappa x)\right] \bar{\phi}_{1}-i \tilde{\alpha}_{e} \kappa \frac{\partial \bar{\phi}_{1}}{\partial \kappa}\right\}+\bar{G}_{0 e}
\end{aligned}
$$

where

$$
\begin{gathered}
\bar{G}_{0 e}=\frac{m v}{e \alpha} \exp \left\{i \frac{m v}{e \alpha}\left[\kappa v \sin \beta-\left(\omega-\kappa V_{0}\right) \beta\right]\right\} \int_{\infty}^{\beta} \bar{g}_{0 e} \\
\times \exp \left\{-i \frac{m v}{e \alpha}\left[\kappa v \sin \beta^{\prime}-\left(\omega-\kappa V_{0}\right) \beta^{\prime}\right]\right\} \\
\bar{g}_{0 e}=\int_{-\lambda}^{\lambda} \int_{0}^{\infty} f_{1}\left(t=0, \mathbf{x}^{\prime}, \mathbf{v}\right) e^{i \omega t-i \kappa \cdot\left(\mathbf{x}^{\prime}-\mathbf{x}\right)} d t d \mathbf{x}^{\prime} \\
\tilde{f}_{0}\left(\mathbf{v}+\mathbf{V}_{0}\right)=\frac{n_{e 0}}{\sqrt{ }(\pi) a} e^{-\left(\mathbf{v}+\nabla_{0}\right)^{2} / a^{2}} \\
\tilde{\alpha}_{e}=\frac{e \alpha}{k T_{e}} \quad \text { and } \quad \tilde{\gamma}_{e}=\frac{e \gamma}{k T_{e}} .
\end{gathered}
$$

When the transforms (3.6) are substituted into (3.4) which, after simplification, becomes

Note that

$$
\kappa^{2} \bar{\varphi}_{1}=4 \pi e\left(\int \bar{F}_{1} d \mathbf{v}-\int \bar{f}_{1} d \mathbf{v}\right)
$$

$$
\int \bar{F}_{1} d \mathbf{v}=\int_{-\infty}^{\infty} d v \int_{0}^{2 \pi} \bar{F}_{1} d \beta
$$

Integrating over $\beta$ space, and noting the orthogonality of Bessel functions we have

$$
\int_{0}^{2 \pi} J n(\xi) J m(\xi) e^{i \beta(m-n)} d \beta= \begin{cases}2 \pi J n^{2}(\xi) & \text { if } m=n \\ 0 & \text { if } m \neq n .\end{cases}
$$

Hence (3.17) becomes

$$
\begin{aligned}
& \int \bar{F}_{1} d \mathbf{v}=2 \pi \frac{e}{M}\left\{\left[\left(1+\tilde{\gamma}_{i}\right) \kappa+\tilde{\alpha}_{i}(-i+\kappa x)\right] \bar{\varphi}_{1}+i \tilde{\alpha}_{i} \kappa \frac{\partial \bar{\varphi}_{1}}{\partial \kappa}\right\} . \\
& \quad \times \sum_{n} \int_{-\alpha}^{\infty} \frac{n e \alpha}{M \kappa \omega^{\prime} v} \frac{1}{v-\frac{n e \alpha}{M \omega^{\prime}}} J n^{2}\left(\frac{M \kappa v^{2}}{e \alpha}\right) \frac{\partial \widetilde{F}_{0}}{\partial v} d v+\int_{-\omega}^{\infty} d v \int_{0}^{2 \pi} \bar{G}_{0 i} d \beta .
\end{aligned}
$$

Substituting (3.13), (3.14) and (3.18) into (3.16), we have

$$
H(\omega, \kappa ; x) \bar{\varphi}_{1}=\psi(\omega, \kappa ; x)
$$


where

$$
\begin{gathered}
\psi(\omega, \kappa ; x)=\frac{4 \pi e}{\omega_{P i}{ }^{2}}\left[\int_{-\infty}^{\infty} d v \int_{0}^{2 \pi} d \beta\left(\tilde{G}_{0 i}-\tilde{G}_{0 e}\right)\right] \\
H(\omega, \kappa ; x)=\frac{\kappa^{2}}{2 \pi \omega_{P i}{ }^{2}}-\left[\left(1+\tilde{\gamma}_{i}\right) \kappa+\tilde{\alpha}_{i}(-i+\kappa x)\right] \sum_{n} \int_{-\infty}^{\infty} \frac{n e \alpha}{M \kappa \omega^{\prime} v} \\
\times \frac{1}{v-\frac{n e \alpha}{M \omega^{\prime}}} J n^{2}\left(\frac{M \kappa v^{2}}{e \alpha}\right) \frac{\partial \widetilde{F}_{0}}{\partial v} d v-\left[\left(1-\tilde{\gamma}_{e}\right) \kappa-\tilde{\alpha}_{e}(-i+\kappa x)\right] \\
\times \sum_{n} \int_{-\infty}^{\infty} \frac{n e \alpha}{m \kappa \omega^{\prime} v} \frac{1}{v-\frac{n e \alpha}{m \omega^{\prime}}} J n^{2}\left(\frac{m \kappa v^{2}}{e \alpha}\right) \frac{\partial \tilde{f}_{0}}{\partial v} d v .
\end{gathered}
$$

In the low frequency case as ion oscillation, the wave vector gradient of the potential $\bar{\varphi}_{1}$ is relatively small, and again the coefficient $\tilde{\alpha}_{i} \kappa$ or $\tilde{\alpha}_{e} \kappa$ is no more greater than the other terms, accordingly the wave vector gradient term is neglected in our simplified model calculation. The validity of this assertion can be verified posteriorly.*

The perturbed potential in time-coordinate of physical space can be shown as (STIX, 1962; JACKSON, 1960)

$$
\bar{\varphi}_{1}(\kappa, t ; x)=\int_{\omega} e^{-i \omega t} \frac{\psi(\omega, \kappa ; x)}{H(\omega, \kappa ; x)} d \omega .
$$

The behavior of time-dependent plasma disturbance shall be determined by the structure of the integrand in (3.22). It is noted that this integrand is made up of two parts-the denominator $H(\omega, \kappa ; x)$ is prescribed in terms of the equilibrium properties of the plasma; the numerator, $\psi(\omega, \kappa ; x)$, the initial perturbations $\bar{g}_{0 i}$ and $\bar{g}_{0 e}$. Since the unperturbed distribution functions for both ions and electrons in velocity space given in (2.6) and (2.7) are single-hump functions, and the initial perturbations $\bar{g}_{0 i},(3.8)$, and $\bar{g}_{0 e},(3.15)$, are entire functions of $v$, considering $v$ as a complex variable (STIX, 1962; JACKson, 1960). The possibility of damping, growing or steady state oscillations of the plasma is governed by the poles of the integrand of (3.22). The numerator, the entire functions of $v$, will not have poles in the complex plane, so the time-dependencies of $\bar{\varphi}_{1}(\kappa, t ; x)$ shall be determined completely by the zeros of $H(\omega, \kappa ; x)$. The equation

$$
H(\omega, \kappa ; x)=0
$$

is often called the dispersion relation since it shows the relation $\omega-\omega(\kappa, x)$ in the solution. Based on these arguments, we have dispersion relation as follows:

$$
\begin{gathered}
\frac{\kappa^{2}}{2 \pi \omega_{P i}{ }^{2}}=\left[\left(1+\tilde{\gamma}_{i}\right) \kappa+\tilde{\alpha}_{i}(-i+\kappa x)\right] \sum_{n} \int_{-\infty}^{\infty} \frac{n e \alpha}{M \kappa \omega^{\prime} v} \frac{1}{v-\frac{n e \alpha}{M \omega^{\prime}}} J n^{2}\left(\frac{M \kappa v^{2}}{e \alpha}\right) \frac{\partial \widetilde{F}_{0}}{\partial v} d v \\
\quad+\left[\left(1-\tilde{\gamma}_{e}\right) \kappa-\tilde{\gamma}_{e}(-i+\kappa x)\right] \sum_{n} \int_{-\infty}^{\infty} \frac{n e \alpha}{m \kappa \omega^{\prime} v} \frac{1}{v-\frac{n e \alpha}{m \omega^{\prime}}} J n^{2}\left(\frac{m \kappa v^{2}}{e \alpha}\right) \frac{\partial \tilde{f}_{0}}{\partial v} d v
\end{gathered}
$$

\footnotetext{
* See Discussion at the end of Section 3.
} 
Note that $\left(M_{\kappa} v^{2} / e \alpha\right) \sim O\left(M v^{2} / e \lambda\right) \sim O\left(M v^{2} / e \varphi_{0}\right)$ where $v=C_{x}-V_{0}$ is the ionic velocity measured with respect to the moving body $\left(V_{0}\right)$ and also that the condition for a particle $(M, v)$ to be trapped in a potential trough $\left(\varphi_{0}\right)$ is $\left(\frac{1}{2} M v^{2}\right) / e \varphi_{0}<1$ (BoHM and Gross, 1949). Consequently the argument $\left(M_{\kappa} v^{2} / e \alpha\right)$ of the Bessel function in equation (3.24) which governs the oscillation characteristics of the trapped ions must comply with the condition $M_{\kappa} v^{2} / e \alpha<1$. Using the small argument expansion for Bessel function (Magnus and OBERHetTinger, 1954)

$$
J n(x) \approx \frac{x^{n}}{z^{n} \Gamma(n+1)}
$$

and also the orthogonality of Bessel functions, (3.24) becomes

$$
\begin{aligned}
& \frac{\kappa^{2}}{2 \pi \omega_{P_{2}}{ }^{2}} \approx\left[\left(1+\tilde{\gamma}_{i}\right) \kappa\right.\left.+\tilde{\alpha}_{i}(-i+\kappa x)\right] \int_{-\infty}^{\infty}\left[\frac{1}{4 \Gamma^{2}(2)} \frac{M \kappa}{e \alpha \omega^{\prime}} \frac{v^{3}}{v-\frac{e \alpha}{M \omega^{\prime}}}+\ldots\right] \\
& \times \frac{\partial \tilde{F}_{0}}{\partial v} d v+\left[\left(1-\tilde{\gamma}_{e}\right) \kappa-\tilde{\alpha}_{e}(-i+\kappa x)\right] \\
& \times \int_{-\infty}^{\infty}\left[\frac{1}{4 \Gamma^{2}(2)} \frac{m \kappa}{e \alpha \omega^{\prime}} \frac{v^{3}}{v-\frac{e \alpha}{m \omega^{\prime}}}+\ldots\right] \cdot \frac{\partial \tilde{f}_{0}}{\partial v} \partial v .
\end{aligned}
$$

Note that $\widetilde{F}_{0}$ and $\tilde{f}_{0}$ are the unperturbed distribution functions, namely Maxwellian or Gaussian; hence the plasma dispersion function, $Z(\zeta)$ can be introduced as follows (FrIEd and Conte, 1961):

$$
Z(\zeta)=\frac{1}{\sqrt{ }(\pi)} \int_{-\infty}^{\infty} \frac{e^{-x^{2}}}{x-\zeta} d x=2 i e^{-\zeta^{2}} \int_{-\infty}^{i \zeta} e^{-t^{2}} d t
$$

This expression is valid for both $\operatorname{Im} \zeta>0$, and $\operatorname{Im} \zeta<0$ provided the analytical continuation concept has been applied (see Appendix). Making use of the relation

$$
\int_{-\infty}^{\infty} \frac{\tilde{f}^{(n)}}{v-\zeta} d v=n ! \int_{-\infty}^{\infty} \frac{\tilde{f}}{(v-\zeta)^{n+1}} d v
$$

the general expressions $I(\zeta)$ for ions and electrons, in terms of the plasma dispersion function are (see Appendix)

$$
\begin{aligned}
& \int_{-\infty}^{\infty} \frac{\tilde{F}_{0}}{(v-\zeta)^{n}} d v=\frac{1}{(n-1) ! A^{n-1}} Z^{(n-1)}\left(\frac{\zeta}{A}\right) \\
& \int_{-\infty}^{\infty} \frac{\tilde{f}_{0}}{(v-\zeta)^{n}} d v=\frac{1}{(n-1) ! A^{n-1}}\left(\frac{\delta}{\theta}\right)^{n / 2} Z\left[\frac{\zeta}{A}\left(\frac{\delta}{\theta}\right)^{1 / 2}\right]
\end{aligned}
$$

respectively. Note that $Z^{(n)}(x)=d^{n} Z / d x^{n} ; \delta=m / M ; \theta=T_{e} / T_{i}$.

The integration of the dispersion relation (3.25) can be performed by the use of 
the expressions (3.28) and (3.29), with terms of the second and higher orders $(n \leq 2)$ truncated,

$$
\begin{aligned}
\frac{\kappa^{2}}{\pi \kappa_{D}{ }^{2}}= & \frac{\kappa A^{2}}{4 \omega_{0} \omega^{\prime}}\left\{[ ( 1 + \tilde { \gamma } _ { i } ) \kappa + \tilde { \alpha } _ { i } ( - i + \kappa x ) ] \left[\sqrt{ }(\pi)\left(\frac{2 V_{0}}{A}-\frac{\omega_{0}}{\omega^{\prime}}\right)\right.\right. \\
& \left.+\left(\frac{\omega_{0}}{\omega^{\prime}}\right)^{3} \quad\left(\frac{\omega_{0}}{\omega^{\prime}}\right)\right]+\left[\left(1-\tilde{\gamma}_{e}\right) \kappa-\tilde{\alpha}_{e}(-i+\kappa x)\right] \\
& \left.\times\left[\sqrt{ }(\pi)\left(\frac{\theta}{\delta}\right)^{1 / 2}\left(\frac{2 V_{0}}{A}-\frac{\omega_{0}}{\omega^{\prime} \delta}\right)+\frac{1}{\delta^{5 / 2} \theta^{1 / 2}}\left(\frac{\omega_{0}}{\omega^{\prime}}\right)^{3} Z^{\prime}\left(\frac{\omega_{0}}{\omega^{\prime}} \delta^{1 / 2} \theta^{-1 / 2}\right)\right]\right\}
\end{aligned}
$$

where $\omega_{0}=e \alpha / M A$.

Notice that the dispersion relation (3.30) depends, among other things, on the Doppler frequency $\omega^{\prime}$ which is related to the particle velocity $V_{0}$ and field frequency $\omega$ via $\omega^{\prime}=\omega-\kappa V_{0}$. When particles are trapped their Doppler frequencies approach zero. Since the oscillations of trapped ion is of interest here, $\omega^{\prime}$ can be herein assumed small. Under this condition the plasma dispersion function can be expanded in the larger argument power series (FRIED and Contre, 1961; FrIED and Gould, 1961) namely

$$
\begin{aligned}
Z(\zeta) \approx i \sqrt{ }(\pi) \exp \left(-\zeta^{2}\right) & -\sum_{n} \frac{\left(-\zeta^{2}\right)^{n} \sqrt{ }(\pi)}{\left(n+\frac{1}{2}\right) !} \\
& \approx i \sqrt{ }(\pi) \exp \left(-\zeta^{2}\right)-\frac{1}{\zeta}\left(1+\frac{1}{2 \zeta^{2}}+\frac{3}{4 \zeta^{4}}+\ldots\right) .
\end{aligned}
$$

Setting $x=0$ in equation (3.30), we obtain the dispersion relation corresponding to the point of minimum potential which, after the substitution of (3.31) and simplification, becomes

$$
\begin{aligned}
\omega^{\prime} & \approx \frac{A \omega_{0}}{2 \sqrt{ }(\pi) V_{0}\left[\left(1+\tilde{\gamma}_{i}\right)+\left(1-\tilde{\gamma}_{e}\right) \theta^{1 / 2} \delta^{-1 / 2}\right]}\left\{\omega _ { 0 } \left[\left(1+\tilde{\gamma}_{i}\right)(1-\sqrt{ }(\pi))\right.\right. \\
& \left.+\theta^{1 / 2} \delta^{-7 / 2}\left(1-\bar{\gamma}_{e}\right)\left(1-\sqrt{ }(\pi) \delta^{2}\right)\right]+\frac{i \tilde{\alpha}_{e} \theta^{1 / 2}}{\kappa \delta^{7 / 2}\left[\left(1+\tilde{\gamma}_{e} \theta\right)+\left(1-\tilde{\gamma}_{e}\right) \theta^{1 / 2} \delta^{-1 / 2}\right]} \\
& \times\left[\left(-\theta^{1 / 2}+\delta^{-1 / 2}\right)\left[\delta^{7 / 2}\left(1+\tilde{\gamma}_{e} \theta\right)(-\sqrt{ }(\pi)+1)+\theta^{1 / 2}\left(1-\tilde{\gamma}_{e}\right)\left(-\sqrt{ }(\pi) \delta^{2}+1\right)\right]\right. \\
& \left.\left.-\left[\delta^{7 / 2} \theta^{1 / 2}(\sqrt{ }(\pi)-1)+\left(1-\sqrt{ }(\pi) \delta^{2}\right)\right]\left[\left(1+\tilde{\gamma}_{e} \theta\right)+\left(1-\tilde{\gamma}_{e}\right) \theta^{1 / 2} \delta^{1 / 2}\right]\right]\right\} .
\end{aligned}
$$

The real part determines the Doppler frequency; the imaginary part, the stability of the oscillations (STIX, 1962): e.g. the condition Im $\omega^{\prime}<0$ implies amplification; Im $\omega^{\prime}>0$, damping; Im $\omega^{\prime}=0$, neutral state. The imaginary part of expression (3.32) can be further simplified by eliminating terms of order $\delta^{7 / 2}$ and beyond.

$$
\operatorname{Im} \omega^{\prime} \approx-\frac{A \theta^{1 / 2} \omega_{0} \tilde{\alpha}_{e}(\theta+1)\left(1-\sqrt{ }(\pi) \delta^{2}\right)}{2 \sqrt{ }(\pi) V_{0} \delta^{7 / 2} \kappa\left[\left(1+\tilde{\gamma}_{e} \theta\right)+\left(1-\tilde{\gamma}_{e}\right) \theta^{1 / 2} \delta^{-1 / 2}\right]}
$$

where $\delta=m / M \ll 1$ and $\tilde{\gamma}_{e}=e^{\gamma} / k T_{e} \ll 1$. 
Consequently, Im $\omega^{\prime}$ always has negative values provided $\alpha \neq 0$ and $\theta \neq 0$. Accordingly, we may conclude that there is amplification for the longitudinal ionic oscillations in a negative finite potential trough, provided the temperature ratio $T_{e} / T_{i} \neq 0$.

The neglect of the wave vector gradient $\left(\partial \bar{\varphi}_{1} / \partial \kappa\right)$ term of equation (3.21) in our simplified model for the determination of the neutral stability contour can be now justified $a$ posteriori. Recall that near the neutral stability curve the condition Im $\omega^{\prime} \rightarrow 0$ prevails. It is seen from the integral (3.22) and the condition (3.23) that near the contour of neutral stability and under the condition $M_{\kappa} v^{2} / e \alpha<1$ the following condition becomes obvious: $\left(\partial \bar{\varphi}_{1} / \partial \kappa\right) / \bar{\varphi}_{1} \rightarrow 0$, as $\operatorname{Im} \omega^{\prime} \rightarrow 0$.

\section{Discussions and Conclusions}

On the basis of the present analysis which is valid for a negative potential trough of small depth, the longitudinal ionic oscillations in the trough appear always observable. This conclusion further confirms the preliminary prediction (LIU, 1967; 1969a) that the low frequency plasma oscillations monitored in the wake of Ariel I Satellite appear to be the longitudinal oscillations of the trapped ions in the potential trough behind a Satellite.

A word of caution concerning the effect of geomagnetic field which has been ignored in the present analysis is in order. The orientation of the magnetic field lines relative to the $x$-axis, along which the present one-dimensional potential trough is aligned, must be taken into account. It can be shown that when the external magnetic field $\mathbf{B}_{0}$ is along $x$, and is parallel to the wave vector $\kappa$ the longitudinal motion is almost the same as in the absence of a magnetic field. This is physically reasonable since the electrons gyrate in many tight spirals during each oscillation along the direction of the magnetic field. The present analysis needs modification when there is a significant component of geomagnetic field normal to the stretch of the potential trough.

The presence of above-mentioned longitudinal oscillations of the trapped ions could be indirectly responsible for the plasma ringing phenomena that have been excited by the Satellite Alouette 1 and other ionospheric probes at the approximate local plasma frequency, upper hybrid frequency and cyclotron frequency harmonics (CALVERT and Goe, 1963). It has been suggested that near these frequencies, the warm plasma electrostatic waves, which could normally carry the energy away, have near zero group velocity. The energy consequently remains in the vicinity of the antenna and manifests itself in the ringing. In view of the results of the present analysis, we would like to offer an alternative hypothesis concerning the source of the plasma ringing phenomena monitored on board of Alouette I and other ionospheric probes.

In view of the non-homogeneity of the plasma and the relative motions between the ions and electrons in the satellite wake, it might be expected that available mechanisms for exciting electron oscillations are not lacking (BUNEMAN, 1958; 1964). We would like to draw particular attention to the finding of transient modification of plasma non-homogeneity by the ionic oscillations in a diode discharge in which there is a maximum concentration of plasma electrons (EMFLEUS and JonEs, 1966). It was found that in the interaction between the oscillations of ions and electrons, the ionic oscillations may cause the maximum and minimum electron concentrations 
to shift in space. The coupling found between electronic and ionic oscillations is also noteworthy (EMELeUs and JoNEs, 1964). It is conceivable that the electron oscillations observed on Alouette I may originate from the satellite wake. When the geomagnetic field is taken into account the other high frequency oscillations monitored on Alouette I can accordingly be interpreted.

Acknowledgement--The present work has been supported by NASA Research Grants NsG-660 and NGR 23-005-094.

\section{ReFERENCES}

Anderson N. and Arthurs A. M.

Bernstern I. B.

$1968 \quad J$. Math. Phys. 9, 2037.

Bernstein I. B., Greene J. M. and

1958 Phys. Rev. 109, 10.

KRUSkat M. D.

BoHm D. and Gross E.

1957 Phys. Rev. 108, 546.

Buneman O.

Bungman O.

Calvert $W$. and Got G. B.

Chapman S. and Cowling T. G.

1949 Phys. Rev. 75, 1851.

1958 Phys. Rev. Lett. 1, 8.

1964 Physics Fluids 7, 53.

$1963 J$ Jeophys. Res. 68, 6113.

1939 The Mathematical Theory of Non-Uniform Gases. Cambridge University Press, London.

Dirac P. A. M.

1947

Emeleus K. G. and Jones J. M.

Emeleus K. G. and Jones J. M.

Fried B. D. and Conte S. D.

1966

1964

The Principle of Quantum Mechanics, 3rd Ed. Oxford University Press, London.

Fried B. D. and Gould R. W.

Ginzburg V. C.

1961 The Plasma Dispersion Function. Academic Press, New York.

1961 Physics Fluids 4, 139.

1961 Propagation of Electromagnetic Waves in Plasma (transl.). Gordon \& Breach, New York.

JACKSON J. D.

1960

$J$. nucl. Energy $C 1,171$.

LaNDaU L. D.

1946

J. Phys. (U.S.S.R) 10, 25.

LIU V. C.

LIU V. C. and Jew H.

$1967 \quad$ Nature, Lond. 215, 127.

1967 Rarefied Gas Dynamics (Edited by C. L. Brundin), Vol. 2, p. 1703. Academic Press, New York.

LIU V. C. and Hung R. J.

1968

LIU V. C.

$1909 \mathrm{a}$ Space Research IX (Edited by K. S. W. Champion, P. A. Smith and R. L. Smrth-Rose), p. 423. North-Holland, Amstordam.

LIU V. C.

Magnus W. and Oberhettinger F.

1969b Space Sci. Rev. 9 (In press).

1954 Formulus and Theorems for the Functions of Mathematical Physics. Chelsen, New York.

Stix T. H.

1962 The Theory of Plasma Waves. McGrawHill, New York.

Van Kampen N. G.

$1955 \quad$ Physica 21, 945.

\section{APPENDIX}

To perform the integration in (3.25), we would like to discuss briefly a method of integration which has been used in evaluating such improper integrals (STIX, 1962; JACksov, 1960). 


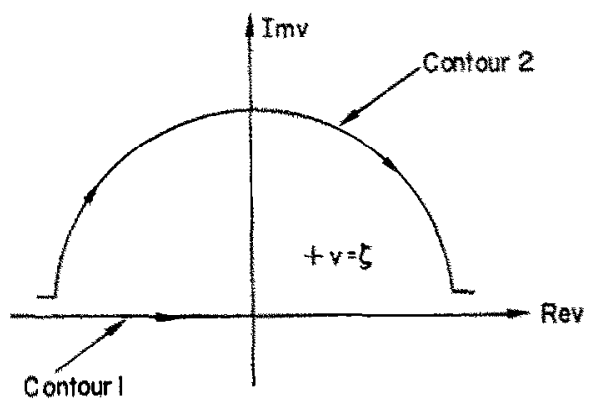

Fig. Al. Integration contours of $I(\zeta)$ with pole at $\operatorname{Im} \zeta>0$.

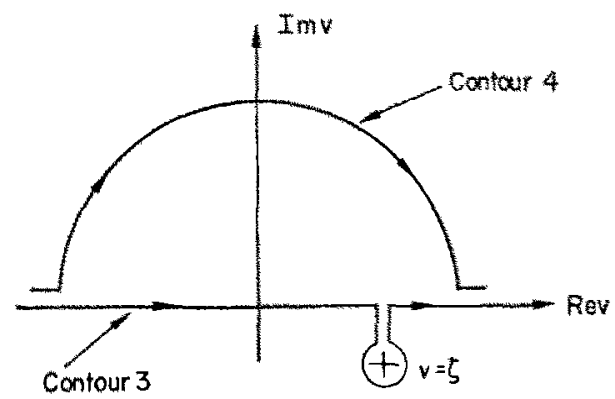

Fig. A2. Deformed contours of $I(\zeta)$ with pole at $\operatorname{Im} \zeta<0$.

Consider the following characteristic integral

$$
I(\zeta)=\int_{-\infty}^{\infty} \frac{f_{(b)}}{v-\xi} d v
$$

Clearly the analytic properties of $I(\zeta)$ depend among other things on those of $f(v)$. Physically, $f(v)$ expresses the unperturbed equilibrium distribution of either electrons or ions as the case may be. Should the given equilibrium distribution be an analytie even function, it will also be an entire function in the complex plane pathological on the real axis. Accordingly. (A.I) can be treated under the following cases:

(1) When Im $\zeta>0$, the prescribed contour along the real axis, namely contour $I$, in Fig. A1, can be replaced by contour 2 , and the integral $I(\zeta)$ can be evaluated as the principal value of the integral

$$
I(\zeta)=P \int_{-\infty}^{\infty} \frac{f(v)}{v-\zeta} d v
$$

(2) When $\operatorname{Im} \zeta<0$, there is a pole in the lower $\zeta$ plane. Since $f(v)$ is an entire function, the concept of analytical continuation can be applied by introducing contour 3 (see Fig. A2) following LaNDAU (1946). Actually the integration of $I(\zeta)$ in (A.I) along contour 3 is equivalent to that along contour 4 plus residue of the pole at $v=\zeta$. It has been shown by VAN KaMres (1955) that

$$
\lim _{\xi \rightarrow+i \varepsilon} \frac{1}{v^{*}-\zeta}=P\left(\frac{1}{v-\zeta}\right)+\pi i \delta(y-\zeta)
$$

where $\varepsilon$ denotes a infinitesimally small value, and $\delta$ denotes Dirac's $\delta$-funetion (Drrac, 1947). The introduction of (A.3) into (A.1) leads to

$$
I(\zeta)=\int_{-\infty}^{\infty}\left[P\left(\frac{1}{v-\dot{\zeta}}\right)+i \pi \delta(v-\zeta)\right] f(v) d v=P \int_{-\infty}^{\infty} \frac{f^{\prime}(v)}{v-\zeta} d v+\pi i f(\zeta)
$$


The expression (A.4) can be generalized to (JACKSON, 1960)

$$
\int_{-\infty}^{\infty} \frac{1}{v-\zeta} \frac{\partial^{n} \vec{f}}{\partial v^{n}} d v=P \int_{-\infty}^{\infty} \frac{f^{(n)}(v)}{v-\zeta} d v+\pi i f^{(n)}(\zeta)
$$

where $f^{(n)}(v)$ denotes the $n$th derivative of $f$ with respect to $v$.

\section{Notation}

$$
\begin{aligned}
& F(x, v, t) \quad \text { ion distribution function } \\
& f(x, v, t) \quad \text { electron distribution function } \\
& M \quad \text { ion mass } \\
& m \quad \text { electron mass } \\
& V_{0} \quad \text { stream velocity } \\
& \phi(x, t) \quad \text { potential distribution } \\
& k \quad \text { Boltzmann constant } \\
& T_{i} \quad \text { ion temperature } \\
& T_{e} \quad \text { electron temperature } \\
& A^{2}=\frac{2 k T_{i}}{M} \\
& a^{2}=\frac{2 k T_{e}}{m} \\
& \lambda_{D}^{2}=\frac{k T_{i}}{4 \pi e^{2} n_{i_{0}}} \\
& \lambda_{d}^{2}=\frac{k T_{e}}{4 \pi e^{2} n_{e 0}} \\
& n_{i} \\
& n_{e} \\
& \kappa \quad \text { wave number } \\
& \text { ion density } \\
& \text { electron density } \\
& \kappa_{D}=\frac{1}{\lambda_{D}} \\
& \kappa_{d}=\frac{1}{\lambda_{d}} \\
& \lambda \\
& \alpha \\
& \gamma \\
& \omega_{p_{i}}{ }^{2}=\frac{4 \pi e^{2} n_{i 0}}{M} \\
& \omega_{p_{\theta}}{ }^{2}=\frac{4 \pi e^{2} n_{e 0}}{m} \\
& \omega_{0}=\frac{\mathrm{e} \alpha}{M A} \\
& \omega^{\prime}=\omega-\kappa V_{0} \\
& \delta=m / M \\
& \theta=T_{e} / T_{i}
\end{aligned}
$$$$
\text { Subscripts }
$$

ion

electron

perturbation state. 